\title{
Reflets
}

Revue ontaroise d'intervention sociale et communautaire

\section{La violence en milieu scolaire : un défi pour intervenants et intervenantes}

\section{Marthe Duhamel-Maples}

Volume 2, numéro 1, printemps 1996

Contrer la violence subie par les femmes et les enfants en milieu familial

URI : https://id.erudit.org/iderudit/026104ar

DOI : https://doi.org/10.7202/026104ar

Aller au sommaire du numéro

\section{Éditeur(s)}

Reflets : Revue ontaroise d'intervention sociale et communautaire

ISSN

1203-4576 (imprimé)

1712-8498 (numérique)

Découvrir la revue

Citer cet article

Duhamel-Maples, M. (1996). La violence en milieu scolaire : un défi pour intervenants et intervenantes. Reflets, 2(1), 40-57.

https://doi.org/10.7202/026104ar
Résumé de l'article

À titre d'intervenante, l'auteure s'attarde à dresser un portrait de la violence en milieu scolaire.Elle présente certains facteurs de risques : géographiques, socio-économiques, culturels,familiaux, scolaires, personnels et interpersonnels. Dans un deuxième temps, l'auteure montreque pour contrer la violence en milieu scolaire, certains programmes ont été mis en place dansla région d'Ottawa-Carleton. L'intervention multidimensionnelle qu'elle propose est fondéesur des mesures de prévention et de médiation.
Tous droits réservés (C) Reflets : Revue ontaroise d'intervention sociale et communautaire, 1996
Ce document est protégé par la loi sur le droit d'auteur. L'utilisation des services d’Érudit (y compris la reproduction) est assujettie à sa politique d'utilisation que vous pouvez consulter en ligne.

https://apropos.erudit.org/fr/usagers/politique-dutilisation/ 


\section{La violence en milieu scolaire:}

\section{un défi pour intervenants et intervenantes}

À titre d'intervenante, l'auteure s'attarde à dresser un portrait de la violence en milieu scolaire. Elle présente certains facteurs de risques: géographiques, socio-économiques, alturels, familiaux, solaires, personnels et interpersonnels. D ans un deuxième temps, l'auteure montre que pour contrer la violence en milieu scolaire, certains programmes ont été mis en place dans la région d' 0 ttawa-C arleton. $L$ 'intervention multidimensionnelle qu'elle propose est fondée sur des mesures de prévention et de médiation.

\section{M arthe D uhamel-M aples}

$C$ hef des services de santé mentale

$\mathrm{C}$ entre psychosodial pour enfants et familles d' 0 ttawa- $\mathrm{C}$ arleton

C 'est à titre d'intervenante en milieu scolaire et communautaire que nous avons constaté à quel point le phénomène de la violence a pénétré l'école, allant jusqu'à perturber sérieusement le processus d'apprentissage qui est la raison d'être de cette institution. N ous avons également entendu les plaintes, les frustrations, les réactions de colère de certains intervenants impuissants devant ce phénomène. Enfin, beaucoup d'enfants agressifs, peu tolérants à l'égard des figures d'autorité, maladroits avec leurs pairs, perturbés et surtout perturbateurs, se sont confiés et nous ont fait part de leur mal de vivre.Tous admettent l'importance de modifier le cours actuel des événements afin de rétablir un climat plus sain à l'école.

Dans cet article, nous présenterons une définition de la violence en milieu scolaire, tenterons de dresser un portrait réaliste 
de la situation, et proposerons certaines explications. Par la suite, nous examinerons certaines initiatives visant à réduire l'agressivité manifeste des jeunes afin de recréer l'école comme milieu propice à l'apprentissage.

\section{Violence et agressivité}

La violence exprime un certain état des rapports sociaux et de ce fait, dépend du contexte social et culturel. Plus précisément, le milieu de vie, propre à une collectivité donnée, détermine les seuils d'antisocialité et de gravité des actes posés par les individus (H ébert, 1991; Vitaro et al. , 1994).

L'agressivité se définit par une disposition mentale. L'agression, pour sa part, représente le passage à l'acte, jugé socialement inacceptable, et comporte un pouvoir, une domination organisée en rapport social ayant pour but ultime de nuire à autrui.

\section{Description des comportements agressifs}

II faut reconnaître qu'une majorité de jeunes ont recours occasionnellement à des comportements agressifs durant leur enfance ou leur adolescence. Leur répertoire de comportements agressifs se compare à celui des adultes, issus d'un milieu social et culturel donné (Hébert, 1991).

Selon les études, les garçons présentent de trois à huit fois plus de comportements agressifs que les filles. C es dernières se démarquent par une agressivité verbale plus aiguisée, tandis que les garçons agressifs montrent un manque de contrôle et une faible tolérance à la frustration. C hez ceux-ci, ces traits de personnalité 
deviennent manifestes à partir de l'âge de dix ans (Vitaro et al., 1994).

O Iweus (1978) distingue, chez les garçons agressifs, deux catégo ries complémentai res: les bagar reurs (bullies), genre de petits caïds qui harcèlent et intimident d'autres garçons, et les victimes (whipping boys), sorte de souffre-douleurs de ces premiers. L'intimidation regroupe des actes agressifs comme l'insulte, la menace, les coups physiques ou toutes autres formes de malaises. C ette forme de violence interpersonnelle se produit assez souvent à l'école.

Les victimes d'intimidation apparaissent comme des «élèves plutôt anxieux, craintifs, sensibles, ayant une estime d'eux-mêmes plutôt faible, et se percevant comme sans intérêt pour les autres» (Vitaro et al., 1994: 36). À l'opposé, les bagarreurs se montrent impulsifs, ont peu de contrôle sur leurs tendances agressives, et témoignent d'une attitude positive face à la violence. D e plus, ils peuvent démontrer un besoin de dominer autrui et n'ont pas une estime de soi négative. $D$ ans la dynamique relationnelle entre ces deux catégories d'enfants, il est intéressant de noter que la victime ne rétorque pas généralement. De plus, elle a peu de chance que d'autres enfants viennent à sa défense, car elle n'a généralement pas le support de ses camarades. Si la victime se confie à un adulte compréhensif, elle redoutera les représailles des bagarreurs.

Un autre élément important est la stabilité des comportements antisociaux. À la différence des enfants dont les comportements antisociaux se résorbent d'eux-mêmes après l'âge de dix ans, on note chez ceux dont les problèmes comportementaux persistent après cet âge, un certain nombre de récurrence.A insi, ces derniers présentent une manifestation plus précoce de problèmes de comportement, liée à plus d'un contexte (famille, école), à un taux plus élevé et selon une variété de formes (Loeber, 1982). $D$ 'après plusieurs études, il semble qu'il y ait une continuité entre les problèmes de comportements à l'âge préscolaire et les difficultés ultérieures d'adaptation. 
«L es enfants de maternelle jugés physiquement agressifs, hyperactifs, et désobéissants affichent plus de problèmes de comportement... à l'âge de dix ans que les enfants ne présentant pas un tel profil de comportement... »
L es enfants de maternelle jugés physiquement agressifs, hyperadifs, et désobéissants affichent plus de problèmes de comportement (agressivité, turbulence, délinquance) à l'âge de dix ans que les enfants ne présentant pas un tel profil de comportement, indique les données recueillies auprès d'un échantillon d'enfants québécois (V itaro et al., 1994:38)

Selon le champ professionnel, diverses appellations désignent ces troubles de comportements. En pédopsychiatrie, il sera question de troubles d'opposition, de troubles de conduite, de déficits d'attention; en travail social, on les abordera sous l'angle de l'antisocialité et de la déviance sociale; en éducation, on parlera de trouble de comportement, de mésadaptation socioaffective, de problèmes de discipline, d'agressivité, d'hyperactivité, de délinquance.

\section{Facteurs de risque et explications}

En psychologie, il est plutôt rare que les recherches convergent vers un modèle unique d'explication du comportement humain. La psychologie, science humaine et sociale, ne peut prétendre à la précision des sciences exactes, car elle cherche à comprendre le comportement individuel en contexte. En ce sens, il serait utopique d'isoler l'individu de son environnement qui lui procure des stimulations et des occasions d'agir. C'est pourquoi nous adhérons à un modèle du développement de l'enfant, qualifié de transactionnel.

Le développement psychosocial futur d'un individu est fonction de ses caractéristiques passées et actuelles, de celles de son environnement (facteurs surtout statutaires), ainsi que de ses expériences futures (facteurs dynamiques). $D$ e plus, certains attributs personnels ont, en partie, été façonnés par son environnement, 
lequel $a$, en retour, été particulièrement modelé par ces

caractéristiques personnelles (V itaro et al., 1994: 39)

D ans les prochaines sections, nous allons distinguer les facteurs de risque liés aux comportements agressifs, pour faciliter la clarté de notre exposé, bien qu'il fasse se souvenir qu'ils sont le plus souvent enchevêtrés l'un, l'autre.

\section{Facteurs géographique, socio-économique, culturel}

Plusieursétudes, au Q uébec (Tremblay, 1994), en 0 ntario (O fford et al., 1986; Boyle et O fford, 1988) et, aux États-U nis (West, 1982), démontrent un taux plus élevé de troubles de comportement parmi les enfants provenant de classes socio-économiques défavorisées, et vivant en milieu urbain. Toutefois, ce facteur de risque est aussi corrélé à d'autres facteurs tels la taille de la famille et le degré de supervision parentale.

\section{Facteurs d'ordre familial}

D es études portant sur des échantillons de garçons agressifs démontrent qu'ils proviennent plus souvent de familles dont les parents sont séparés ou de familles recomposées. O n note aussi parmi ces garçons agressifs une prépondérance d'enfants uniques qui, devenus adolescents, présenteront des problèmes de comportement. Enfin, la fréquentation d'une garderie avant l'entrée en maternelle est associée, à la maternelle, à la présence de comportements agressifs et turbulents (Tremblay et al. , 1990).

C ertains facteurs constituent des éléments de stress ayant un impact sur le comportement des enfants, bien qu'ilsne contribuent

$\ll \ldots$ la discorde maritale constitue un fadeur ayant un effet plus direct et perceptible sur les problèmes de comportement de l'enfant. » pas directement aux difficultés d'adaptation des enfants agressifs. Le climat familial, en terme de présence ou disponibilité parentale, peut être influencé par le statut socio-économique ou la structure familiale. Par exemple, des contraintes budgétaires imposées aux parents accroîtront leur niveau de stress, et affecteront en retour le climat familial. D e même, la discorde maritale constitue un facteur ayant un effet plus direct et perceptible sur les problèmes de comportement de l'enfant. 
«... une gestion de dasse, adoptant une approche punitive et contrôlante, est assodiée à l'apparition de problèmes de comportement, et ce, par opposition à une approche visant I'autonomie et le renforcement de |'étudiant. 》
Par ex emple, le divorce des parents et les problèmes de comportement chez l'enfant sont reliés en vertu du stress qu'imposent par la famille, la discorde maritale et les procédures de divorce, la non-disponibilité affective des parents et leurs pratiques disciplinaires relâchées (V itaro et al., 1994:41)

Ces derniers éléments jouent un rôle crucial dans le développement de l'enfant.

De nombreuses études démontrent également que des caractéristiques, telles l'alcoolisme paternel ou les conduites criminelles du père, sont fortement reliées aux troubles comportementaux de l'enfant. De plus, certaines recherches portant précisément sur les interactions familiales mettent en évidence des échanges négatifs caractérisés par l'escalade du contrôle coercitif (Vitaro et al., 1994). II semble aussi que les parents d'enfants agressifs ne soient pas portés à exercer une supervision étroite des activités de leurs enfants (Vitaro et al., 1994:4).

Enfin, les parents d'enfants ayant des problèmes de comportement éprouvent plus de difficultés personnelles et interpersonnelles à communiquer entre eux, dans une relation conflictuelle. Les enfants, témoins de leurs conversations, captent des messages de nature hostile qu'ils s'approprient, et retransmettent, à l'école, à leurs professeurs et leurs pairs.

\section{Facteurs liés à l'école}

En milieu scolaire, les principales variables pouvant contribuer à l'explication du phénomène de la violence chez les élèves sont les lieux surpeuplés et délabrés, les règlesarbitraireset incohérentes, le mépris de la part des enseignants et des pairs, l'observation et la valorisation de comportements agressifs (H ébert, 1991). Plus particulièrement, une gestion de classe, adoptant une approche punitive et contrôlante, est associée à l'apparition de problèmes de comportement, et ce, par opposition à une approche visant l'autonomie et le renforcement de l'étudiant. Enfin, le rejet par 
les pairs se révèle un prédicteur fiable des difficultés d'adaptation à l'adolescence et à l'âge adulte (Vitaro et al., 1994).

\section{Facteurs d'ordre personnel et interpersonnel}

Le développement du comportement antisocial chez l'enfant tient à plusieurs facteurs tels les prédispositions caractérielles, le style parental, et le déficit dans le comportement. Ce dernier facteur s'inscrit dans le prolongement du rejet des enfants agressifs par leurs pairs et par les enseignants en raison de leurs difficultés d'adaptation dans le jeu.

En effet, les enfants agressifs affichent des lacunes importantes au plan sociocognitif, touchant notamment l'interprétation des situations sociales, la résolution de problèmes et la maitrise de soi. Par exemple, ils ont du mal à percevoir convenablement les intentions d'autrui, et ont tendance à exagérer l'hostilité des autres. Ils ont également tendance à recourir aux solutions violentes sans envisager préalablement une diversité de possibilités. Ils sont portés à réagir sans réfléchir, impulsivement, sans anticiper les conséquences de leur action, et sans prendre connaissance des informations disponibles avant de porter un jugement. Par ailleurs, la plupart des enfants agressifs éprouvent des difficultés scolaires relatives aux troubles d'attention et d'apprentissage. Au plan affectif, les enfants agressifs ont tendance à être déprimés, solitaires, à témoigner peu d'empathie sociale, et à attribuer l'échec de leurs relations interpersonnelles à des événements indépendants de leur volonté (H undert et Burlak, 1991).

C escaractéristiques personnelles et interpersonnelles de l'enfant agressif prennent une nouvelle forme à l'adolescence, par I'ajout de facteurs inhérents à ce stade de développement. D ans le cours normal de son développement, l'adolescent fait face à des remaniements majeurs: crise d'identité, sexualisation, changements des relations avec ses parents et ses amis, changements cognitifs. Cette période de vie se caractérise par une multitude de changements susceptibles de réactiver et d'amplifier des réactions agressives contre autrui, ou contre soi. 
«... plus forte sera l'intégration sociale, moins il y aura de suicide, d'homicide, de délinquance, et de toxicomanie»
L'intensité de ses transformations dans ses rapports à soi et au monde peuvent engendrer des sentiments de privation. A fin d'y pallier, l'adolescent est alors tenté de se réfugier auprès de gang qui sont plus violents aujourd'hui, que ceux d'il y a vingt ans. $C$ 'est pourquoi les recherches des intervenants sociaux mettent l'emphase sur le contrôle social comme élément explicatif. Pour eux, plus forte sera l'intégration sociale, moins il y aura de suicide, d'homicide, de délinquance, et de toxicomanie. Parallèlement, plusieurs recherches indiquent que les changements structuraux, affectant la famille au cours des dernières décennies, ont contribué à un affaiblissement des niveaux d'intégration sociale. À cela s'ajoute également l'absence du père, l'hostilité de la mère, la mésentente, etc., comme autant de facteurs dont il faut tenir compte (C usson, 1991).

II faut cependant préciser que l'activité délinquante n'est pas le propre de l'adolescent perturbé. La majorité des adolescents commettent des actes pouvant être qualifiés de délits (C usson, 1989). Seule une minorité d'entre eux s'engagera plus à fond dans la voie du crime.

À l'opposé de cette violence dirigée vers autrui, il y a la violence tournée vers soi. Le nombre de dépression adolescente est alarmant et les tentatives de suicide vont en augmentant, à tel point qu'on parle même de crise de suicidité chez les adolescents (Baron, 1993).

À l'adolescence, l'individu perturbateur, étiqueté tel en raison de ses caractéristiques agressives, ne perçoit plus l'univers scolaire comme répondant à ses besoins. II prend du retard au plan scolaire, s'absente de l'école, est indiscipliné et désintéressé. C ertaines études montrent que les élèves sous-doués sont surreprésentés parmi les délinquants. $C$ ette explication sommaire se double d'une nouvelle dimension à la lumière de l'immigration. Les jeunes immigrants vivent une situation conflictuelle d'identité. D étachés de leur culture traditionnelle, attirés par les cultures d'accueil dont ils ne font pas réellement parti, ils sont doublement exclus (H ébert, 1991). 


\section{Valeurs sociales}

Auteur du livre $L$ a violence à l'école, $H$ ébert indique que les valeurs de la société, véhiculées par les médias, jouent un rôle important auprès des enfants, en particulier, ceux qui n'ont pas su développer un cadre conceptuel leur permettant d'exercer leur sens critique.

... les formes de violence commercialisées dans plusieurs jeux et sports individuels et collectifs, ainsi que la fré quence de scènes d' agression dans les médias suggèrent que ces voies représentent des réponses efficaces pour gérer des conflits. À long terme, cette médiatisation presque quotidienne de la violence a pour effet de la banaliser. L'ade agressif deviendrait, en dernière instance, un moyen normal de régler un différend ou d'obtenir ce que l'on désire (H ébert, 1991: 39).

«L es conduites violentes chez les jeunes ne sont pas un phénomène nouveau. ... depuis quelques années, elles atteignent "un seuil audelà duquel la qualité de la vie scolaire est compromise " »
À cet égard, l'état d'inachèvement de l'appareil psychique chez l'enfant ou l'adolescent ne lui permet guère de prendre conscience de la distance entre la réalité et l'imaginaire, et ainsi de pouvoir poser un jugement critique.

En résumé, une multiplicité des facteurs agissent simultanément dans l'émergence et la stabilisation des comportements agressifs chez l'enfant et l'adolescent : personnalité du jeune, arrière-plan familial, milieu scolaire. Les conduites violentes chez les jeunes ne sont pas un phénomène nouveau. Pourtant, il semble que, depuis quelques années, elles atteignent «un seuil au-delà duquel la qualité de la vie scolaire est compromise» (C usson, 1990). La principale conséquence de cette violence est I'instauration d'un climat de peur qui afflige autant les élèves que les enseignants, et qui affecte la mission de l'école.

\section{Initiatives en milieu scolaire}

A fin de contrer la violence en milieu scolaire, l'intervention doit 
«... plusieurs conseils scolaires ont adopté une politique de Tolérance zéro face à la violence en milieu scolaire» être multidimensionnelle: communautaire, scolaire et familiale. Au plan scolaire, les programmes doivent rejoindre aussi bien l'enseignant que l'étudiant. Et les interventions doivent lier les aspects psychologique, éducatif, administratif, légal et physique (A pter et Goldstein, 1986). D e fait, la concertation et les efforts de toutes les personnes touchées par la violence doivent être mis à contribution afin de créer un milieu scolaire propice à un apprentissage pacifique.

Au cours des dernières années, plusieurs conseils scolaires ont adopté une politique de Tolérance z éro face à la violence en milieu scolaire Leur succèsà ce chapitre ne se dément pas. C ette politique repose sur un encadrement vigilant, permettant un suivi et une continuité d'intervention de la part de tous les partenai res: enfant, parent, enseignant, administrateur scolaire, intervenant social ou intervenante sociale, et, policier, le cas échéant. U ne telle politique ne saurait être instaurée, sans qu'au préalable, des mesures de prévention soient mises sur pied. C es dernières doivent être mises en place très tôt, au niveau préscolaire, car les comportements agressifs se cristallisent entre six et dix ans, comme le mentionne Tremblay (Tremblay et al., 1994).

\section{Ateliers d'habiletés parentales}

Les relations entre parents et enfants jouent un rôle important dans l'émergence des comportements agressifs chez ces derniers. Le climat hostile, voire inconsistant, dans les relations entre parents et enfants, contribue à l'apparition de l'agressivité chez l'enfant. Les parents incarnent les premiers modèles des enfants. C'est à eux qu'incombe la tâche de transmettre leurs valeurs. Les ateliers d'habiletés parentales s'inscrivent dans cet effort de rejoindre les parents qui vivent quotidiennement avec leurs enfants et qui ressentent le besoin de développer davantage leurs compétences.

Depuisquelques années, le C entre psychosocial offre une série de dix ateliers de trois heures chacun, ayant pour but d'amener I'individu à être bien dans sa peau, et développer avec les autres des relations autonomes et responsables. Plus précisément, les objectifs poursuivis sont: développer sa confiance en soi comme 
«L'agressivité de nos enfants nous ramène sans cesse à la nôtre» individu et parent, augmenter son estime de soi, acquérir des habiletés et des savoir-faire comme parent, et enfin, éprouver un sentiment de compétence parentale. En somme, il s'agit de donner aux parents un forum dans lequel ils peuvent discuter librement et ouvertement de leurs difficultés, de leur offrir des moyens afin de réagir adéquatement dans telle ou telle situation, d'acquérir des moyens autres que la punition, et développer une meilleure communication avec leur enfant. II s'agit donc d'un cheminement en dix étapes où la première sera consacrée à un retour sur sa propre enfance. $D$ ans une analyse transactionnelle où coexiste le parent, l'enfant et l'adulte dans chaque individu, ce retour permet aux parents de prendre contact avec l'enfant qui existe en eux, afin d'être mieux en mesure d'appréhender le vécu de leur enfant. $D$ ans les étapes ultérieures, les parents sont invités à prendre conscience de leurs propres besoins comme individu, avant d'aborder la discipline proprement dite. Enfin, les dernières étapes de ce processus portent sur les techniques de communication, soit l'écoute active, l'affirmation de soi, la négociation et la résolution de conflits. De plus, le programme de dix ateliers vise à sensibiliser les participants aux obstacles pouvant nuire à une communication saine et entière.

U ne telle approche rejoint indirectement l'enfant agressif en lui offrant un autre modèle parental. C ela permet alors à l'enfant de développer sa capacité à accepter un délai entre son émotion et le passage à l'acte, à éprouver de l'empathie, à rechercher des solutions à ses problèmes de manière constructive et positive, et enfin, à canaliser l'expression de sa colère d'une manière acceptable.

L'agressivité de nos enfants nous ramène sans cesse à la nôtre II y a des familles où, de génération en géné ration, la violence se perpétue jusqu'à ce qu' un individu ait le courage de travailler suffisamment sur lui pour épargner ses propres enfants (L aporte, $1990: 45$ ).

\section{Programmes d'habiletés sociales}

Les enfants ayant un comportement antisocial, nous l'avons vu, ne possèdent pas les compétences sociales nécessaires, pour être 
acceptés de leur camarades et permettre des relations amicales. D e nombreux programmes d'habiletés sociales visent justement à combler ces lacunes et susciter l'acquisition d'aptitudes sociales, axées sur la résolution de problèmes interpersonnels (réflexion), les comportements sociaux (bonne conduite) et l'identification des sentiments (affectivité).

Ces divers programmes datent déjà d'un certain temps et étaient destinés, à l'origine, aux enfants présentant des comportements agressifs. $O \mathrm{r}$, cette approche très ciblée comportait l'inconvénient majeur de ne pas encourager la général isation des acquis en milieu naturel. C'est pourquoi, au cours des dernières années, cette méthode fut étendue à la classe entière et présente ainsi plusieurs avantages.

Elle permet au personnel enseignant de développer des stratégies propres à contrer l'indiscipline de certains enfants dans leur propre classe. Les enfants ayant des problèmes de comportement ne se sentent plus stigmatisés puisque toute la classe participe au programme. Ainsi, les enfants agressifs ont donc moins d'occasions de mettre en pratique certains comportements antisociaux appris, puisqu'ilsne sont plus l'objet du rejet de leurs pairs. II devient alors plus facile aux enseignants de trouver les moyens d'encourager des comportements sociaux acceptables. La plupart des programmes disponibles visent une clientèle âgée de six à dix ans, car c'est à ce moment que l'enfant développe graduellement les capacités cognitives nécessaires à la résolution de conflits. C ela dit, il existe certains programmes s'adressant à une clientèle préscolaire (Goldstein, 1990) ou adolescente (PAR C).

Le Centre psychosocial offre deux programmes d'habiletés

«L'objectif général de ces programmes est de réduire la fréquence des comportements inadaptés de l'enfant et d'augmenter ses capadités de contrôle sur ses comportements. » sociales pour les six à douze ans. L'un est d'origine ontarienne, et fait partie d'un projet trimestriel de recherche dirigé par I'université M cM aster (H undert et Burlak, 1991); I'autre est d'origine québécoise, connu sous le nom de PAR C, et vise à développer des aptitudes d'auto-contrôle, de résolution de problèmes et de compétence sociale.

L'objectif général de ces programmes est de réduire la fréquence des comportements inadaptés de l'enfant et d'augmenter ses capacités de contrôle sur ses comportements. Parallèlement à 
«... la médiation, comme méthode d'intervention, a connu un essor sans précédent, et ce, dans les litiges familiaux... et les conflits entre enfants en milieu scolaire... » cette diminution, on tente de leur substituer des comportements prosociaux, susceptibles de recevoir dans l'entourage de l'enfant, une réponse positive. $D$ e plus, ces programmescherchent à inclure les parents à la démarche, en leur consacrant un volet bien à eux, et ce, dans le but qu'ils adoptent une attitude et une pratique éducatives capables de concourir à la réussite de l'objectif général des programmes mentionnés ci-dessus.

C es ateliers d'habiletés sociales s'appuient sur une approche behaviorale-cognitive. C elle-ci reconnaît à la fois l'importance des facteurs environnementaux dans l'émergence et le maintien des comportements, et le rôle de la personne comme agent de changement, à savoir que la personne est capable d'exercer une maîtrise cognitive de ses émotions.

... selon cette approche, une relation existe entre les cognitions (ou les idées) et les émotions: ainsi, la façon dont une personne étiquette ou évalue une situation sur la base de ses attentes et de ses croyances, affecte ses réactions émotionnelles face à cette situation (P otvin et al., 1994).

En pratique, ces ateliers sont consacrés à l'acquisition spécifique de comportements ayant trait à la résolution de problèmes, la gestion de la colère, l'identification des émotions, etc. Chaque atelier dure quarante minutes, et comporte des misesen situation, des jeux de rôles, des simulations, l'apprentissage par modelage, et des discussions. II y a un atelier par semaine. D ans l'intervalle, l'enseignant ou l'enseignante cherche à renforcer positivement les comportements visés. Ainsi, les enfants apprennent graduellement à s'observer et à s'évaluer, ce qui les responsabilise face à leur comportement.

\section{Programmes de médiation}

Au cours des dernières années, la médiation, comme méthode d'intervention, a connu un essor sans précédent, et ce, dans les litiges familiaux (médiation familiale), et les conflits entre enfants en milieu scolaire (médiation par les pairs). 
La médiation est un processus volontaire qui vise à fadiliter le dialogue entre deux personnes en conflit. U ne tierce personne aide les parties à résoudre ellesmêmes leur conflit d'une façon mutuellement avantageuse (T itley, 1993:13).

Les conflits qu'ils soient d'ordre personnel, familial, national ou international, meublent le quotidien de tous et chacun. $0 \mathrm{r}$, on peut regrouper les réponses au conflit en trois catégories: évitement, confrontation, communication. L'évitement comporte le retrait, l'ignorance ou la négation de la situation conflictuelle; la confrontation recouvre l'expression de menaces, d'agression ou de colère dans un conflit; la communication ouvre à la compréhension et au respect mutuel en vue de permettre la résolution du conflit (Schrumpf et al., 1991). La médiation par les pairs promeut, évidemment, la communication comme réponse à une situation conflictuelle et permet la recherche d'objectifscommuns.

Contrairement à la confrontation où l'énergie dépensée par les participants est dirigée l'un contre l'autre, la communication favorise une dépense énergétique offrant des possibilités d'expression et d'acceptation des sentiments de chacun des participants, de clarification des faits, d'écoute active et d'intégration des points de vue.

Le processus de médiation comprend trois personnes. Les deux parties adverses et une tierce personne agissant comme médiateur ou médiatrice. Les étapes de la médiation sont l'ouverture où la médiatrice précise les buts de la médiation; la cueillette de renseignements où chacune des parties offre sa version des faits; le rapprochement où on met l'accent sur les intérêts communs et les points d'accord; la solution où l'on procède à l'identification et à l'évaluation des options possibles afin d'adopter une solution concertée; la fermeture où I'on procède à la rédaction de l'entente sur la solution mutuellement envisagée (Schrumpf, 1991).

U ne médiation est réussie lorsque les parties adverses sont parvenues à débroussailler et améliorer leurs relations, à satisfaire leurs préoccupations, à jeter les fondements de leur relation future et à maintenir le caractère privé de leur dispute. Elles auront 
«L a médiation... permet de faire face à la violence en milieu scolaire et d'atteindre des objectifs à plus long terme, contrairement aux approches coeratives... qui n'offrent que des solutions à court terme.» alors su développer une plus grande confiance, une meilleure compréhension et un respect mutuel (C habot, 1994).

La médiation, comme toutes les approches centrées sur le développement de la personne, permet de faire face à la violence en milieu scolaire et d'atteindre des objectifsà plus long terme, contrairement aux approches coercitives (expulsion, suspension) qui n'offrent que des solutions à court terme. Trop souvent, l'emploi de mesures coercitives radicales répond aux besoins du système, de l'administration scolaire, et ne s'attaque pas au fond du problème.

\section{Philosophie globale de gestion}

S'ils ne reçoivent pas l'assentiment de tout le personnel ou s'ils ne sont que partiellement appliqués, les programmes décrits cidessus n'obtiendront que des réussites mitigées. À cet égard, l'ensemble du milieu scolaire a un rôle vital à jouer. II importe que tous soient sensibilisés à la prévention de la violence. L'école se doit d'adopter une position claire et consistante de façon à favoriser l'émergence d'un climat propice à la résolution des conflits. De la direction à la conciergerie, le personnel de l'école doit, par leur propre conduite, donner l'exemple, et offrir aux jeunes des modèles dignes d'être admirés et adoptés.

... l'école doit offrir aux jeunes des modèles de comportement «pro-social». Tant la direction que le personnel enseignant doivent faire preuve de respect et de tolérance dans leur interaction avec les élèves de façon à favoriser le développement de la confiance et de l'estime de soi chez eux (T itley, 1993).

\section{Programme de jour, en milieu externe}

Le $C$ entre psychosocial, conjointement avec le $C$ onseil des écoles publiques d' $\mathrm{O}$ ttawa- $\mathrm{C}$ arleton, offre un programme de jour à l'enfant qui ne parvient plusà fonctionner dans son école de quartier, 
à cause de sa violence ou de ses comportements inadaptés. A insi, le $C$ entre dispense des services thérapeutiques à une clientèle dont l'âge varie entre quatre et vingt-et-un ans, se répartissant selon différents niveaux scolaires.

Les groupes sont constitués d'un nombre limité d'enfants. C es derniers bénéficient de la présence et du soutien d'un grand nombre d'adultes: personnel scolaire, éducateurs ou éducatrices spécialisées. Les services thérapeutiques visent la réinsertion sociale et scolaire du jeune, en prise à de sérieux problèmes d'adaptation. Les services offerts entourant la démarche de l'enfant vont de la thérapie individuelle ou familiale au counselling parental ou à un suivi comportemental assidu et constant. 0 n emploie couramment des techniques de modification du comportement, alors que des ateliers d'habiletés sociales sont offerts une fois la semaine. Les parents sont invités à participer avec le personnel affecté aux plans de traitement du programme, à la rédaction de bilans réguliers et à l'orientation de la thérapie de l'enfant.

Un service résidentiel est aussi disponible pour les enfants dont les difficultés familiales entravent leur démarche d'adaptation globale. Parfois, c'est à la demande des parents que l'enfant sera mis en résidence, afin de leur permettre de récupérer leur capacité de gestion parentale.

\section{Conclusion}

La violence des enfants nous interroge dans notre rôle d'intervenants et d'intervenantes. II faut obtenir pour eux ce que la vie, jusqu'à présent, n'a pas su leur apporter : d'être entouré de deux parents aimants et disponibles, d'avoir des relations significatives et de qualité avec des adultes complaisants et compréhensifs, de prendre des moyens pacifiques pour régler les querelles et les différends.

C 'est pourquoi nous avons besoin d' éducateurs, mais aussi d'idéaux et de projets de vie donnant un sens à 
la relation éducative. Le caractère massif des actes de violence révèle un dysfonđionnement de l'éducation. L es enfants ne naissent pas en ayant le sens de la loi. Ils ne savent pas IIs ont besoin d'apprendre par I'intermédiaire d'adultes (A natrella, 1995).

II est donc de notre ressort de participer à la prévention des problèmes de violence dès la petite enfance, période où l'enfant nous offre un bien précieux, sa confiance. Sachonsprendre comme objectif d'orienter les tout-petits vers la plénitude du savoir être, afin qu'ils puissent disposer, un jour, du savoir faire indispensable à leur adaptation sociale.

\section{Bibliographie}

AN AT R ELLA, Tony (1995). «La violence- transgression» dansL a violence chez les jeunes, compréhension et intervention, (ouvrage collectif sous la direction deY von L'A bbé), M ontréal, Éditions Sciences et Culture.

APTER, S.et J. GO LDSTEIN (1986). Youth V iolence, Programs \& Prospeds, Pergamon Press, N ew York.

BAR O N , Pierre (1993). La dépression chez les adolescents, O ttawa, Edisem - M aloine.

CH ABOT, M - F. (1994). «Prévention de la violence dans les écoles», M ontréal, U niversité M cGill, $C$ entre de perfectionnement du leadership en éducation, Séminaires pour éducateurs hors pairs.

C U SSO N , M . (1989). D élinquants: Pourquoi?, M ontréal, Bibliothèque québécoise.

C U SSO N , M . (1990). «La violence à l'école: le problème et les solutions», A pprentissage et socialisation, vol.13, no.3, septembre, 213-221.

HÉBERT, J. (1991). L a violence à l'école, M ontréal, Éditions Logiques.

HUNDERT, J. et P. BU R LAK (1991). G uide de l'animateur pour le programme de développement des aptitudes sodales en dasse, U niversité M cM aster, (document pour diffusion restreinte).

LAPO RTE, D. et al., (1990). D u coté des enfants I, M ontréal, Hôpital Ste-Justine.

LOEBER , R . (1982). «T he stability of antisocial and delinquent child behavior. A review», C hild D evelopment, 53, 1431-1446.

M CGIN N IS, E. et A.P. GOLDSTEIN (1990). Skill Streaming in E arly C hildhood, R esearch Press Company, Illinois.

H O SER , G. (1987). L'agression, Paris, Presses universitaires de France.

O LW EU S, D.(1978). A gression in the schools: bullies and whipping boys, Washington, D.C., H emisphere.

SCHRU M PF, F. et al. (1991). Per M ediation, C onflict R esolution in schools, Illinois, R esearch Press Company.

TIT LEY, F. (1993). «La violence à l'école: place à la prévention», Le D roit, 0 ttawa-H ull, p. 13. 
TR EM BLAY, R . (1995). «Les enfants violents à l'école primaire: qui sont-ils et que deviennentils?», dans $L$ a violence chez les jeunes, M ontréal, les Éditions Sciences et C ulture, (ouvrage collectif sous la direction deY von L'A bbé).

TR EM BLAY, R .E. et al. (1990). «La violence physique chez les garçons: un comportement à comprendre et à prévenir», Interface, 11, 12-18.

VITAR O, F., P.L.D O BKIN, C.GAGN ON, et M. LEBLAN C (1994). Les problèmes d'adaptation psychosociale chez l'enfant et l'adolescent: prévalent, déterminants et prévention, Ste-Foy, Presses de I'université du Q uébec.

W EST, D.J. (1982). D elinquency: its roots, careers and prospects, Cambridge $\mathrm{H}$ arvard U niversity Press. 